\title{
What is the best intraocular lens?
}

The best intraocular lens is the natural human lens. It is permanent, maintenance free, focuses automatically, and comes already fitted. It has no aberrations and allows the retinal surgeon an unhampered view of the peripheral retina. As long as the only treatment for a diseased lens is its removal there will be a quest for a prosthesis capable of mimicking the original ever more closely. Among the options considered up to now was the anterior chamber angle fixated lens which was more a compromise of technology and technique than the result of careful research into intraocular lens (IOL) design, and the iris fixated lens which was clearly, in retrospect, a deviation from progressive IOL development. Real progress took place when lens fixation moved back into the posterior chamber and after a period of sulcus fixation it now seems that bag fixation offers huge benefits, not the least of which is the sequestration of the prosthesis. ${ }^{1}$ In addition, bag fixation in a capsule opened by capsulorhexis permits precise placement of the IOL and, consequently, accurate prediction of the postoperative anterior chamber depth. This permits more precise IOL power calculation ${ }^{2}$ and because contemporary wound management techniques avoid iatrogenic astigmatism an experienced hand can aspire to achieve a postoperative refraction within 1 dioptre of emmetropia. In presbyopia, patients who receive a monofocal lens could regard themselves as restored to the visual state they would have enjoyed if they had never developed cataract. Such an outcome could be regarded, even in the narrowest of terms, as a cure. Younger patients invariably lament the loss of accommodation.

In this issue of the journal Williamson et al discuss the performance of monofocal and multifocal lenses. The natural lens accommodates and is not multifocal. The retina is designed to process a single sharply focused image. A multifocal lens presents the retina with an image in which all objects at all distances are both focused and defocused. The subject pays attention to the focused image of interest and ignores all others, both focused and defocused. While the theory seems implausible the practice appears to satisfy the desire for clear vision at all distances. Patients do see well for both near and distance. ${ }^{3}$ But because this is not accommodation there is a compromise and that compromise is the reduction of contrast sensitivity and colour edge definition. But the huge majority of patients selected for multifocal implants seem to have no difficulty with the quality of vision they achieve postoperatively. However, these patients may be comparing multifocal vision with the cataractous vision that led them to surgery in the first place or with clear but presbyopic vision in the fellow eye. I feel sure that when multifocal vision is eventually compared with true accommodation we will find that multifocal vision is inferior in quality. Nevertheless, it could be that our current population of patients with cataract may well prefer multifocal vision to monofocal vision while ophthalmology awaits the development of a truly accommodating lens.

The technological obstacles to such a lens are very great. It now seems that the Helmholtz theory of accommodation does not explain all we now know about the process. Part of accommodation is accounted for by depth of field and tolerance of blur and, in the case of reading, practice at recognising words. Presbyopia is not accounted for simply by sclerosis of the lens nucleus. Those cataract surgeons who operate on all age groups from the very young to the very old will know that not only does the consistency of the lens body change with aging but an equally marked change takes place in the elasticity of the capsule. There is evidence ${ }^{4}$ that the reduced elasticity of the choroid in the region of the ciliary muscle which takes place with aging significantly impairs the forward movement of the ciliary body during accommodation. These three ingredients possibly combine to produce the phenomenon of presbyopia. Consequently, it is not likely that the answer to finding an accommodating prosthesis will be simply finding an implant material with the consistency of young lens fibres. The final solution is likely to require a paradigm shift.

Meanwhile prosthetic lens development will continue and two very promising avenues are being enthusiastically investigated. The first is surface modification and the second is folding lens technology. Heparin surface modification is now established as giving greater biocompatibility ${ }^{5}$ and the new generation of acrylic lenses coated with collagen promise to give enhanced biocompatibility with foldability. Toric IOLs have become a realistic option now that capsulorhexis gives such predictability of fixation and orientation. With the arrival of videokeratoscopy pre-existing corneal astigmatism can be effectively neutralised with an accuracy which, up to now, was not possible. The results of independent clinical trials on these lenses will be eagerly evaluated.

The use of these lenses depends on the presence of an intact capsule. But what is the best IOL for a patient who at the time of implantation has no or insufficient capsule support? Recent advances in technique allow these patients to have a posterior chamber IOL sutured to the ciliary sulcus. The initial success of this technique seems to indicate that this option offers safer IOL fixation and a more comfortable eye although postoperative $\mathrm{AC}$ depth is so far very difficult to predict. We await the results of comparative studies to see how effective is this new modality in terms of visual rehabilitation, endothelial cell survival, and retinal pathology.

Currently, the IOL of first choice is one which is safely placed in the capsular bag through a capsulorhexis opening. It conforms to the dimensions and shape of the bag and is bicompatible with the intraocular environment. Folding lenses offer further advantages both in terms of minimal peroperative invasion and postoperative astigmatic neutrality, and multifocality shows promise as a substitute for accommodation. There is no 'best' intraocular lens but rather there is a set of lenses which offers optimum opportunity for best vision for a particular surgeon's patients. One surgeon's choice of, say, haptic contour may complement his/her technique while the same lens may hamper another's. Bag sized lenses fit the normal eye snugly but are too small for the larger myopic eye. There are different situations where some lenses are more appropriate than others and the well equipped ophthalmic operating room will have several banks of lens styles so that the best lens for each patient is always available.

The research and development of new lens materials and styles is an extremely costly undertaking. Patients rightly demand greater safety in clinical trials and the costs involved in getting a new lens to market run into many millions of pounds. If patients want new IOLs which mimic the natural lens ever more closely then society must resource the research programmes necessary for their development. As this cost increases the question of how to fund this research may need to be reconsidered. Up to now manufacturers have recovered their costs on the sale of the successful lens and this method has clearly been very satisfactory in bringing us this far. However, the paradigm shift needed for a truly accommodating small incision lens may be so costly that no one 
manufacturer could fund it. The question then will be - will anyone?

JOHN BOLGER

Queen Elizabeth II Hospital Howlands, Welwyn Garden City, Herts AL7 4HQ
1 Wasserman D, Apple DJ, Caseneda VE, Tsai JC, Morgan RC, Assia EI. Anterior capsule tears and loop fixation of the posterior chamber lens. Ophthalmology 1991; 98: 425-31.

2 Olsen T, Gimbel H. Phacoemulsification, capsulorhexis and intraocular lens power prediction accuracy. 7 Cataract Refract Surg 1993; 19: 695-9.

3 power prediction accuracy. Cataract Refract Surg 1993, 19:695-9. functional results in patients with multifocal intraocular lenses: a long-term

4 Wyatt $\mathrm{HJ}$. Application of a simple mechanical model of accommodation of the yatt HJ. Application of a simple mech
aging eye. Vis Res 1993; 33: 731-8.

5 Umezawa S, Shimizu K. Biocompatibility of surface-modification of intraocular lenses. $\mathcal{F}$ Cataract Refract Surg 1992; 19: 3714.

\section{Indexing British Fournal of Ophthalmology}

From 1994 there will be a change in the method of indexing the subjects in the British fournal of Ophthalmology. Papers will be indexed by a keyword system, and authors will be asked to choose up to three keywords, occasionally four, for each paper at manuscript stage, which will then contribute to the compilation of an annual index. A keyword is a word (or phrase) which will identify the subject matter of a written paper in an index. The index will be published, as usual, at the end of the December issue. The format will be different, with the title of the paper repeated after each keyword on every entry.

The author index will no longer include the title of the paper but will become a list of authors only with page numbers.

On the surface, choosing index headings would not appear difficult but medicine, because of its synonyms, phrasal headings, trade names and generic names of drugs, syndromes, Latin words, and assorted lay terms, produces problems of its own.

An index should be consistent. It is not good if half the entries are under 'Open angle glaucoma' and the other half under 'Glaucoma, open angle', and, whereas it is reasonable to make some decisions about the entries that can be anticipated, authors will not know what other work is being published, or under what titles, in the same volume and some modifications may have to be made by the technical editor.

Authors are advised to scan papers for keywords that may not be in the title, to use British approved names rather than trade names for drugs, and to avoid general terms such as clinical, complications, adverse effects, and patient. As the subject of the journal is 'ophthalmology' it is better not to use this as a heading with a few exceptions. In general, it is better not to split accepted concepts. For instance, 'Giant cell arteritis' is preferable as a keyword, rather than 'Arteritis, giant cell'.

Some shortened forms can be accepted; DNA, AIDS, HIV, and cAMP are universally known and are the images that spring to mind before looking up, but mostly the full form should be used as the keyword. Mostly, the Greek letter is not used in alphabetisation. Put all veins and arteries under the specific description - for instance, 'Central retinal artery/ vein' under 'Central'. There will be no cross references in the keyword index.

This cannot be more than an attempt to guide authors and perhaps to warn of the pitfalls, but it is important to remember that if authors wish to have their work found easily in the index they should consider the keywords carefully. 